Vol. 14, $n^{\circ} 1 \mid 2010$

Varia

\title{
Defois (Serge), Les avocats nantais au XX siècle
}

Rennes, Presses Universitaires de Rennes, 2007, 397 pp. , ISBN 978

275350522 3, préface de Jean-Claude Farcy, pp. 9-13; postface de Jean

Danet pp. 343-347.

\section{Françoise Briegel}

\section{OpenEdition}

\section{Journals}

Édition électronique

URL : https://journals.openedition.org/chs/1168

DOI : 10.4000/chs. 1168

ISSN : 1663-4837

\section{Éditeur}

Librairie Droz

\section{Édition imprimée}

Date de publication : 1 mai 2010

Pagination : 116-118

ISBN : 978-2-600-01425-0

ISSN : $1422-0857$

Référence électronique

Françoise Briegel, « Defois (Serge), Les avocats nantais au XXe siècle 》, Crime, Histoire \& Sociétés / Crime, History \& Societies [En ligne], Vol. 14, n¹ | 2010, mis en ligne le 07 juin 2010, consulté le 23 mars 2022. URL : http://journals.openedition.org/chs/1168; DOI : https://doi.org/10.4000/chs.1168 
l'assistance judiciaire qui était prise en charge par les confréries, au siècle précédent. À ces valeurs fondatrices d'une identité collective professionnelle s'ajoute le rejet d'autres telles que l'avarice et l'avidité.

La période 1787-1804 voit disparaitre la profession d'avocat. L'engagement de ces derniers dans la rédaction des cahiers de doléances, leur participation aux États Généraux essoufflent leur mobilisation qui était très importante surtout depuis 1787. Afin d'abaisser les coûts de la justice et de permettre au citoyen de se défendre, la réforme de 1790 fait disparaître la profession d'avocat. Dès ce moment et sans manifester de résistance, les avocats voient de nouvelles opportunités s'offrir à eux, puisqu'ils intègrent massivement la magistrature. Malgré le rétablissement du titre en 1804, Hervé Leuwers constate la rupture révolutionnaire qui laisse les membres isolés et les barreaux déstructurés, même si quelques expériences de réorganisation spontanée s'observent sur le territoire.

Dès 1810 et surtout en 1822, les avocats profitent de nouvelles réglementations pour engager leur restructuration. Ils désignent des conseils de discipline, conjointement composés de magistrats et d'avocats, et les barreaux se dotent de bâtonniers. La définition normative à l'échelle du pays, d'un lexique commun (bâtonnier, conseil de discipline) et la clarification des rapports avec la magistrature augurent de la renaissance de la vie professionnelle. La mobilisation commune dans différents barreaux de province, comme sous l'Ancien Régime pour défendre des causes jouent aussi un rôle unificateur. L'Ordonnance de 1830 entérine les exigences du barreau formulées depuis de nombreuses années (la liberté et l'indépendance, l'élargissement et la généralisation des droits individuels et collectifs). Ainsi, le mouvement législatif des années 1804-1830 achève l'homogénéisation structurelle du barreau français à l'échelle nationale. Les activités de défense au civil et au pénal deviennent le monopole des avocats. Cette spécialisation professionnelle et l'interdiction de la double activité, constitutive de la profession d'avocat sous l'Ancien Régime, l'uniformisation des études, l'apprentissage de la culture de la profession grâce aux stages, les consultations gratuites, les conseils, la défense du pauvre, attestent d'une continuité de fonction et de valeurs entre l'Ancien Régime et le XIX ${ }^{\mathrm{e}}$ siècle. Toutefois, l'élaboration d'une culture commune où l'honneur disparaît au profit de la dignité, le renoncement à des revendications traditionnelles du type des privilèges consacre, à partir des années 1820, la profession libérale des avocats.

Françoise Briegel

Université de Genève, Visiting scholar, Center for the Study of Law and Society, Berkeley University Francoise.Briegel@unige.ch

\section{Defois (Serge), Les avocats nantais au XX $X^{e}$ siècle, Rennes, Presses Universitaires de Rennes, 2007, 397 pp., ISBN 978275350522 3, préface de Jean-Claude Farcy, pp. 9-13; postface de Jean Danet pp. 343-347.}

Serge Defois propose une étude en quatre parties sur le barreau nantais entre 1897 et 1976. À partir d'une approche prosopographique qui veut «déconstruire l'identité collective que constitue le barreau » pour « retrouver les individus » et reconstituer le lien social (p. 23), il se propose d'appréhender le groupe socioprofessionnel des avocats nantais qui est constitué d'environ 500 membres pour la période. 
Pour ce faire, sa recherche s'appuie sur des sources écrites (fonds privés, archives de l'ordre, etc.), complétées d'un corpus de sources orales qui sont principalement des entretiens avec des membres du barreau.

Dans une première partie, Serge Defois décrit l'engagement politique et social des avocats qu'il conjugue à l'évolution du recrutement, afin de saisir si le barreau nantais manifeste d'une dynamique ou s'il tend à se replier. Au XIX ${ }^{\mathrm{e}}$ siècle, alors que le rayonnement est assez marqué sur plan national, notamment car les avocats nantais s'impliquent dans la politique auprès des conservateurs de droite de tendance catholique et monarchiste, Serge Defois constate qu'après le premier tiers du $\mathrm{XX}^{\mathrm{e}}$ siècle, l'investissement individuel se modifie. Dès lors, les avocats privilégient une activité associative et culturelle locale. Quant au recrutement, une diminution, surtout après la Deuxième Guerre mondiale s'observe, alors qu'une augmentation des membres, amorcée dans l'entre-deux-guerres, ouvrait sur la féminisation et de rajeunissement du barreau. Dès les années 1970, le rajeunissement du barreau nantais confère un nouveau souffle à la profession qui était «sclérosée » et attachée aux traditions anciennes.

La deuxième partie porte sur l'origine socioprofessionnelle des avocats. La population constitue un groupe assez homogène sur la période étudiée. La grande majorité des avocats est issue des classes dominantes locales. Le modèle dynastique au cœur du recrutement qui reproduit l'hérédité sociale se double de stratégies matrimoniales qui favorisent l'entrée de la moyenne et de la haute bourgeoisie au sein du barreau. D'une manière générale, l'accès des couches populaires représente moins de $10 \%$ des nouveaux avocats qui restent donc très minorisés jusqu'après les années 1960. Ensuite, Serge Defois évoque un «frémissement» de démocratisation de l'accès au barreau, alors que le taux de renouvellement des effectifs tend à diminuer. Cette absence de démocratisation et cette faible féminisation (qui représente néanmoins 10\% de l'effectif entre 1897 et 1976), signalent que le barreau nantais se «recroqueville». De plus, les principales responsabilités hiérarchiques de l'ordre incombent surtout aux «héritiers». Le frein que représente le coût de la formation d'avocat intervient, sans doute, dans la faible démocratisation du recrutement.

La troisième partie consacrée à la formation et à la carrière des avocats, signale combien les conditions de formation au métier d'avocat nécessitent une solide assise financière. En effet, le stage dont la durée varie entre 3 et 5 ans, est faiblement rémunéré. Les carrières sont en général assez courtes; elles s'allongent après les années 1960. L'activité professionnelle devient en effet, plus lucrative à partir de cette période. Ces conditions de formation et la capacité à vivre des honoraires entravent l'ouverture de la profession à certaines catégories socioprofessionnelles.

Finalement, dans la quatrième partie, l'auteur, à partir de la structuration du barreau et d'une approche de la déontologie, montre que l'indépendance et l'autonomisation du barreau se renforcent. La concurrence avec les avoués, dans un premier temps, puis le rattachement des deux professions en 1971 procède d'une défense «du territoire professionnel» qui structure le barreau et renforce son identité collective. Finalement, dans ce processus d'ampleur nationale, le barreau nantais est moteur dans la grève de 1976 qui préside à la formation d'une entité professionnelle à l'échelle nationale: l'organisation nationale des associations d'avocats. Ce mouvement atteste d'une tentative de renforcer la visibilité de cette profession.

La recherche très fouillée de Serge Defois offre l'opportunité de questionner les méthodes et les postulats adoptés dans bon nombre de recherches sur les groupes socioprofessionnels. Comme le suggère Simona Cerruti, la tentative d'appréhender des catégories socioprofessionnelles ne doit pas se limiter à celle qui ne s'occupe 
que de leurs intérêts professionnels ${ }^{8}$. Les analyses socio-historiques s'enrichiraient à élargir l'investigation aux pratiques et dans le cas précis de l'étude de Serge Defois aux pratiques de défenses ou de conseils judiciaires, comme il le propose dans sa courte partie sur l'activité de défense durant la période d'occupation de 1940 à 1944 . Par exemple, la démarche visant à produire une description, par ailleurs implicitement normative, de l'évolution du barreau nantais en terme de «dynamisme» ou de «repli» pourrait se compléter par une mesure de l'«efficacité» professionnelle des avocats. Les procès gagnés, la recherche de sources attestant d'une satisfaction des justiciables ou celles indiquant un sentiment de justice qu'aurait généré chez les parties le travail de conseil seraient des outils également pertinents et novateurs pour appréhender le «rayonnement» du barreau à partir d'éléments exogènes.

Françoise Briegel

Université de Genève, Visiting scholar, Center for the Study of Law and Society, Berkeley University Francoise.Briegel@unige.ch

\section{Galeotti (Mark), Organised Crime in History, London, New York, Routledge, 2008, 190 pp., ISBN 9780415478175.}

The popular press is full of stories about the threat from organised crime - from the Mafia (the word that has become synonymous with organised crime), from the Russian Mafia, Columbian drugs cartels, Chinese Triads, Japanese Yakuza, Jamaican Yardies and so on. Criminal justice professionals co-operate and collaborate against it; politicians proclaim it a major threat and indeed in 2003 the United Nations ratified a Convention on Transnational Organised Crime. It is declared to be a modern phenomenon. Even respectable criminologists in the United States situated its origins back no further than the southern European immigrants, especially Italians and above all Sicilians, who crossed the Atlantic in the late nineteenth and early twentieth centuries brining their own, often violent systems of entrepreneurship.

In this collection Mark Galeotti and the authors that he has assembled set out to challenge the accepted wisdom. In his introduction Galeotti suggests, as a working definition: 'Organised crime is a continuing enterprise, apart from traditional and legal social structures, within which a number of persons work together under their own hierarchy to gain power and profit for their private gain, through illegal activities' (p. 6). The idea that such crime is always 'apart from traditional and legal social structures' might be debated as tending to exclude some corporate duplicity and systemic offences by functionaries of the state, sometimes with the state's blessing. Nevertheless the argument that underpins the collection, that any state or social structure can be the setting for continuing criminal enterprise with the perpetrators working within their own hierarchies, is well made. Generally speaking the essays support the case, though Wisam Mansour's jeu d'esprit on the tale of Ali Baba and the 40 thieves as an allusion to crime in medieval Baghdad is pushing the limits. So too is Frances Berdan's study of crime in pre-conquest Mexico, which asserts that the Aztec

Simona Cerruti, «La construction des catégories sociales », in Jean Boutier, Dominique Julia (eds), Passés recomposés, Champs et Chantiers de l'Histoire, Paris, Éd. Autrement, 1995, pp. 224-242. 